\title{
USE OF CHLOROQUINE IN SHOCK
}

\section{Christopher E. Famewo, "William H. Noble, and M. Bernadette Garvey}

MANY STUDiEs have been done by different investigators on drugs that affect platelet adhesion and aggregation. The ideal drug should be readily available, rapidly effective, relatively cheap, and should not cause increased bleeding. This study was done as a continuation of the search for drugs that inhibit platelet aggregation, so reducing the increase in pulmonary vascular resistance and lung water following haemorrhagic shock. This paper reports our results with Chloroquine, an antimalarial drug.

\section{Methons}

Two similar groups of mongrel dogs were studied. There were five dogs in each group. Anaesthesia was induced with intravenous pentobarbitone $30 \mathrm{mg} / \mathrm{Kg}$, after which one group received intramuscular chloroquine $200 \mathrm{mg}$ and the other group served as a control.

\section{Catheterization}

Pulmonary artery (PA), femoral artery, left atrium (LA) and central venous (CVP) catheters were passed and their positions were confirmed by pressure monitoring. The catheters were kept patent by small regular flushings with normal saline. Heparin was not used.

\section{Format of experiment}

The experiment was divided into four stages (a) pre-shock, (b) shock, (c) retransfusion, and (d) bicarbonate administration.

Shock was induced in both groups by bleeding the dogs to a mean arterial pressure between 40 and $50 \mathrm{~mm} \mathrm{Hg}$. The pre-shock mean pressure was between 100 and $120 \mathrm{~mm} \mathrm{Hg}$. The onset of bleeding was usually about one and a half hours after intramuscular injection of chloroquine $200 \mathrm{mg}$. in the group that received this drug. The dogs were kept in shock for two hours and then re-infused with their own blood, which had been collected into ACD bags. The mortality in shock was about 50 per cent in both groups.

During the four stages of the experiment, the following measurements were made: (1) mean arterial pressure (MAP), (2) central venous pressure (CVP), (3) left atrial (LA) pressure, (4) pulmonary artery (PA) pressure, (5) cardiac output $(\dot{Q}),(6)$ lung water $\left(\mathrm{ETV}_{\mathrm{L}}\right),(7)$ blood gases (arterial and mixed venous), (8) mixed expired $\mathrm{O}_{2}$ and $\mathrm{CO}_{2},(9)$ Chloroquine blood level, and (10) complete

Departments of Anaesthesia and Haematology, St. Michael's Hospital and University of Toronto, Toronto, Ontario, Canada.

${ }^{\circ}$ Dr. C.E. Famewo is now at the University College Hospital, Ibadan, Nigeria. 
coagulation screen. Platelet aggregation was measured by a Payton aggregometer and a Bausch \& Lomb recorder. The lung water and cardiac output were determined by a thermodilution technique. ${ }^{1}$

From the measurements, pulmonary vascular resistance (PVR) and systemic vascular resistance (SVR) were calculated as shown below.

$$
\begin{aligned}
& \text { PVR }=\frac{(\mathrm{PA}-\mathrm{LA}) \times 1332}{\dot{Q}} \text { dynes sec } \mathrm{cm}^{-5} \\
& \mathrm{SVR}=\frac{(\text { MAP }-\mathrm{CVP}) \times 1332}{\dot{Q}} \text { dynes sec } \mathrm{cm}^{-5}
\end{aligned}
$$

The ratio of physiological dead space to tidal volume (VD/VT), and shunt (QS/ QT) were calculated using Duncalf' ${ }^{2}$ method of computer analysis.

\section{Results}

\section{Peripheral Vascular Resistance}

During the shock period PVR rose markedly in both groups, 700 per cent in the control and 500 per cent in the chloroquine group. When blood was re-infused the PVR fell to about 100 per cent above the pre-shock value in the control group and about 50 per cent above the pre-shock value in the chloroquine group (Figure 1). This difference is statistically significant. (Results were considered statistically

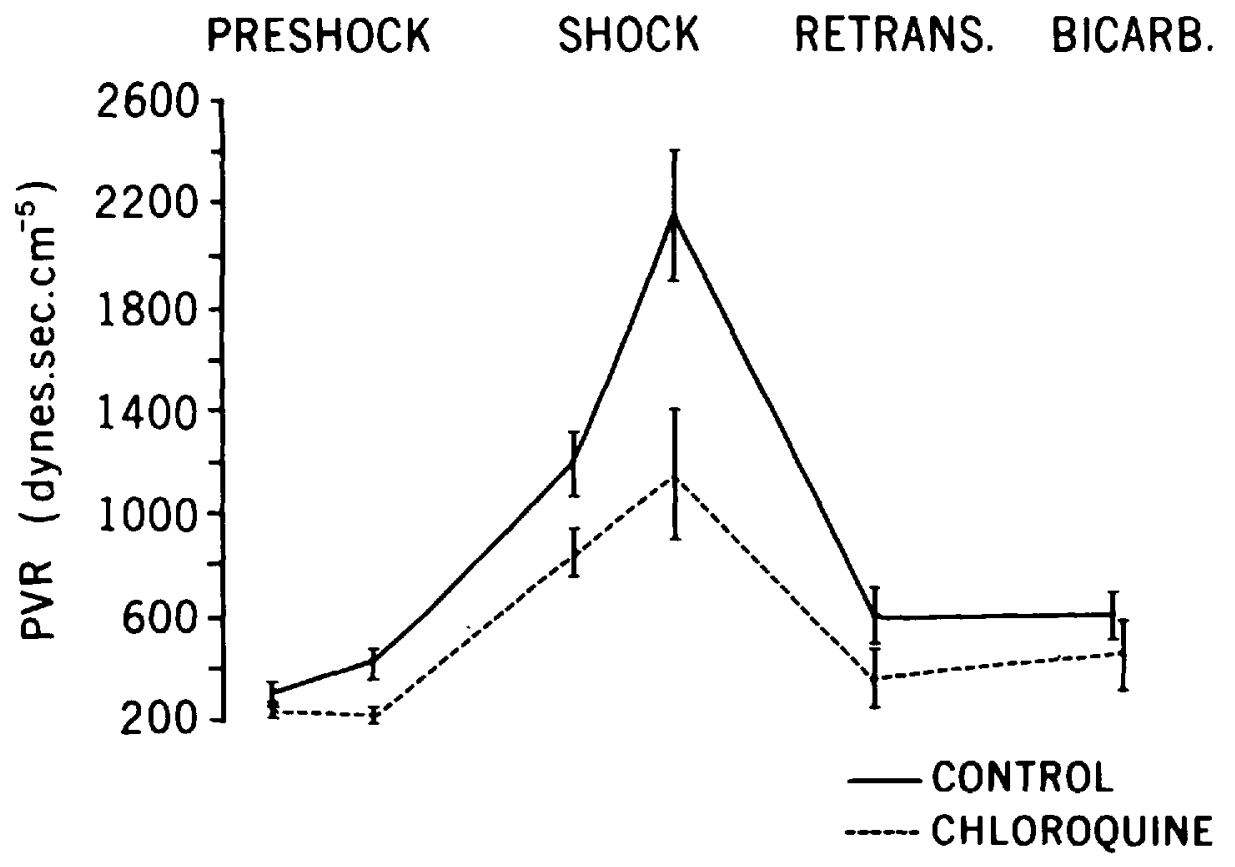

Frcure 1. Changes in Pulmonary Vascular Resistance (PVR) in and out of shock. The stages of the experiment are indicated. Retrans. is after blood retransfusion, bicarb. is after bicarbonate administration to correct the metabolic acidosis of shock. 
significant if $\mathrm{p}<0.01$.) The PVR was not further affected after correction of metabolic acidosis by bicarbonate, indicating that acidosis was not the cause of the elevated PVR. The changes in the base excess at the corresponding stages of the experiment are shown in Figure 2.

\section{Lung Water}

ETV $_{\mathrm{L}}$ fell during shock in both groups. After retransfusion there was a mean increase of 25 per cent in the control but 10 per cent in the chloroquine group above the pre-shock values (Figure 3 ). The difference between the two groups is not statistically significant. Bicarbonate administration resulted in no further change, again excluding the role of acidosis.

\section{Pulmonary Artery Pressure}

PA pressure fell during shock in both groups, but rose above pre-shock value after blood retransfusion. The mean increase was 40 per cent in the control group

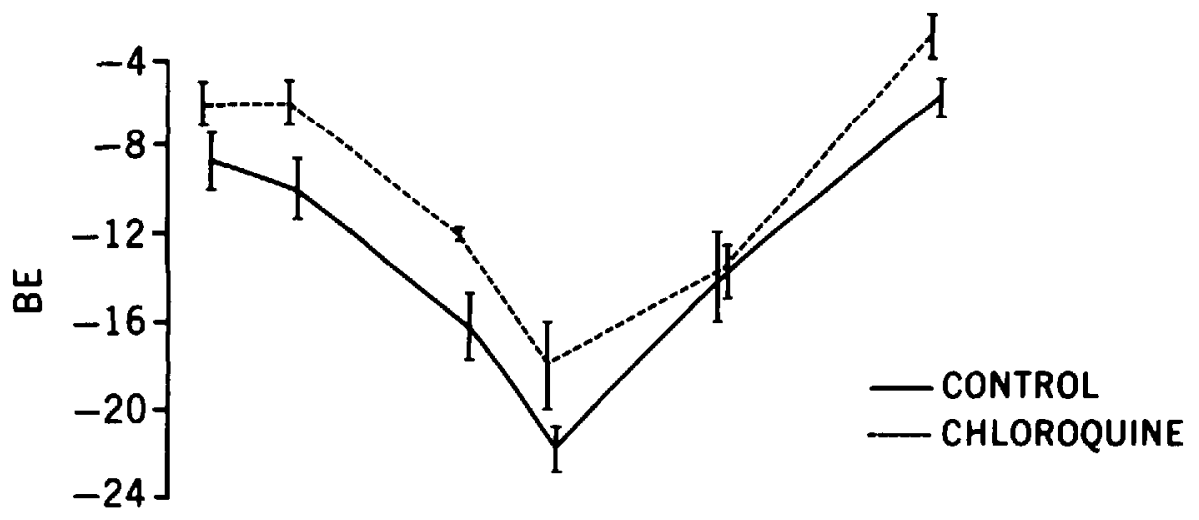

FIGURE 2. Changes in Base Excess (BE) in and out of shock.

\section{PRESHOCK SHOCK RETRANS.}
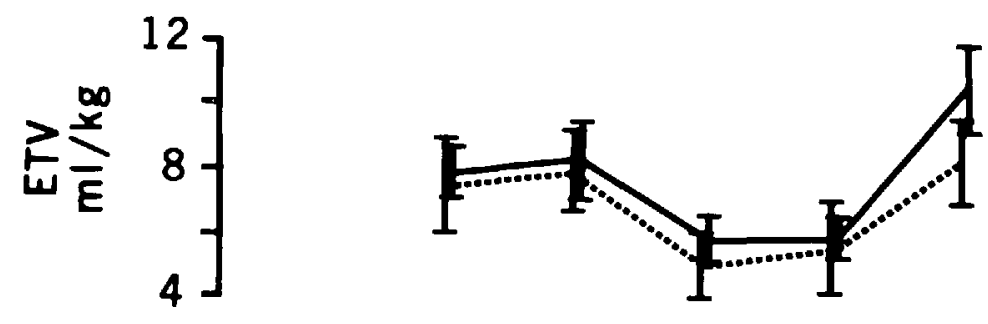

- CONTROL

CHLOROQUINE

Figure 3. Changes in Lung Water $\left(\mathrm{ETV}_{\mathrm{I}}\right)$ in and out of shock. There was 25 per cent elevation in the control dogs, but 10 per cent in the chloroquine-treated dogs after retransfusion of blood. 
and 30 per cent in the chloroquine group. Bicarbonate administration to correct the metabolic acidosis reduced the PA to pre-shock value in the chloroquine group, but was still elevated by 33 per cent in the control group (Figure 4). The difference is statistically significant.
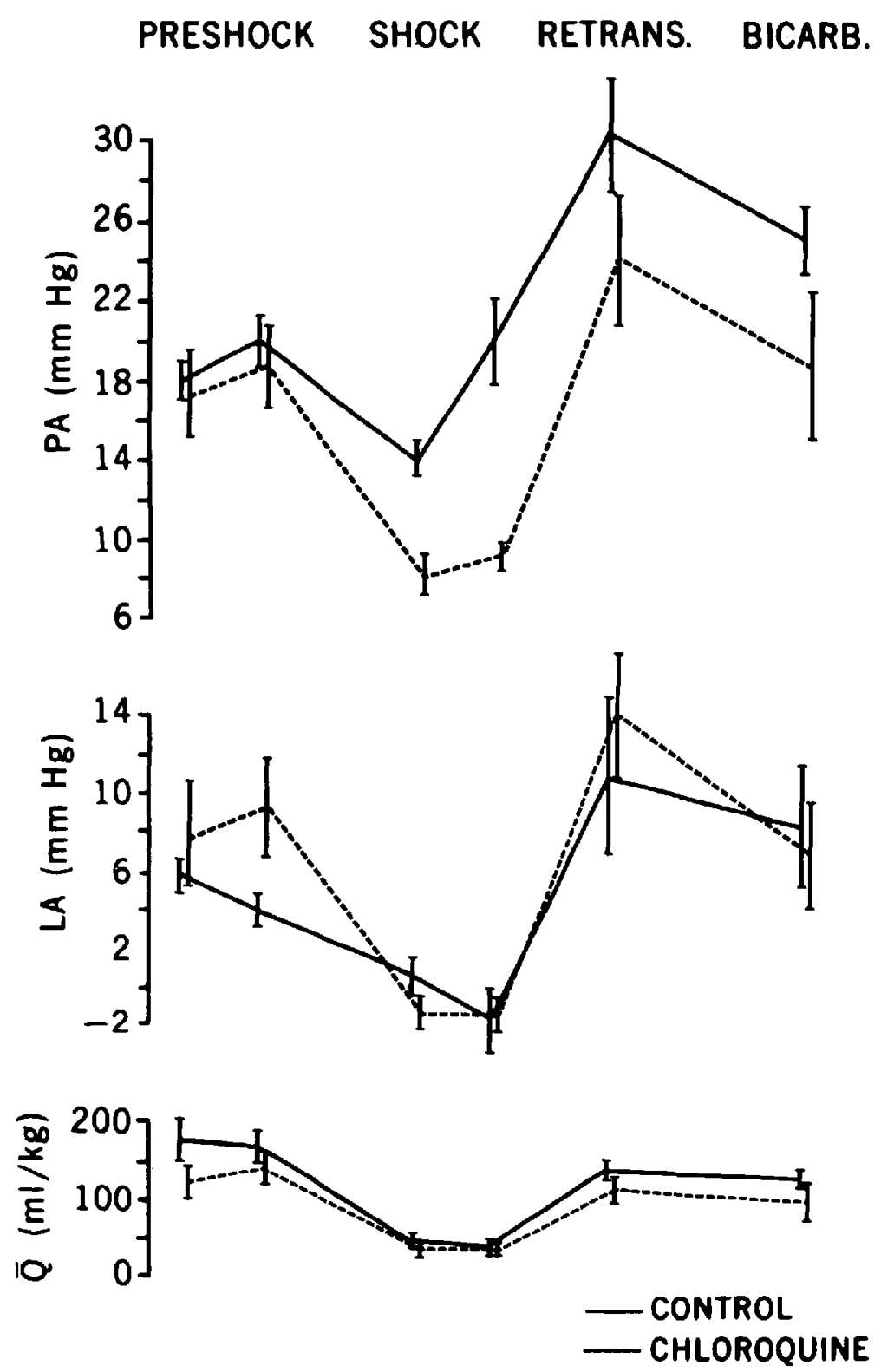

Figure 4. Changes in Pulmonary Artery Pressure in and out of shock. After correction of metabolic acidosis, PA pressures returned to pre-shock value in chloroquine-treated dogs but were elevated by 33 per cent in control. LA Pressure changes in and out of shock similar in both control and chloroquine-treated dogs. Cardiac output changes in and out of shock similar in both control and chloroquine-treated dogs. 


\section{Cardiac Output}

Q fell during shock and rose to near the pre-shock value after retransfusion. Changes in and out of shock were parallel in both groups (Figure 4).

\section{Left Atrial Pressure}

LA pressure fell during shock but rose to above the pre-shock value after retransfusion in a similar fashion in both groups. Bicarbonate administration resulted in a reduction in the LA pressure in both groups but not back to the pre-shock values (Figure 4 ).

\section{Coagulation Studies}

In vitro platelet aggregation was reduced by 50 per cent after chloroquine administration (Figure 5). This correlated with the finding of less platelet loss

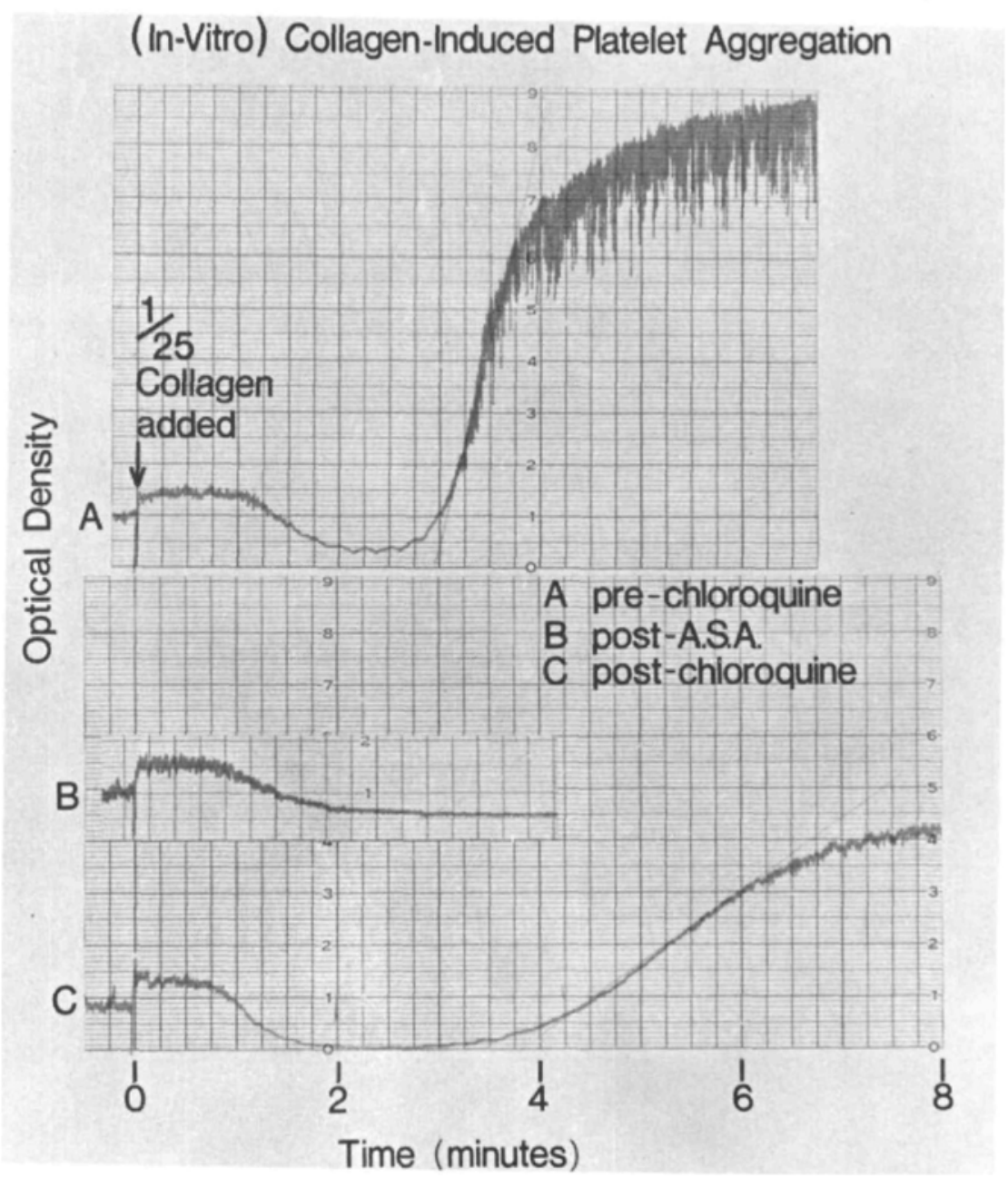

FIGURE 5. A comparison of the effect of chloroquine and aspirin on in vitro collagen induced platelet aggregation. Chloroquine reduced platelet aggregation by 50 per cent and aspirin reduced it by 100 per cent. 
during shock in the chloroquine-treated dogs (Figure 6). The chloroquine blood levels were very small, in the range of 2 to $7 \mu \mathrm{g} \%$. The prothrombin time and partial thromboplastin time were prolonged and fibrinogen degradation products (FDP) were present, suggesting disseminated intravascular coagulation (DIC) in both groups.

The changes in the blood gases (Figure 7), shunt and dead space (Figure 8) in both groups of dogs during and after shock are shown.

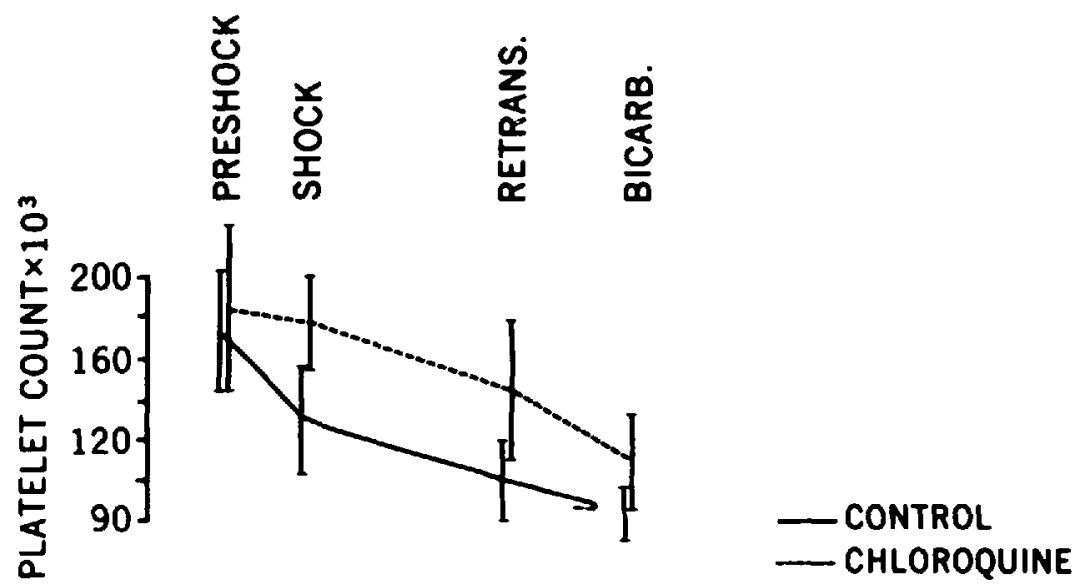

Figure 6. Platelet count in and out of shock. Platelet loss is less marked in chloroquine-treated dogs.
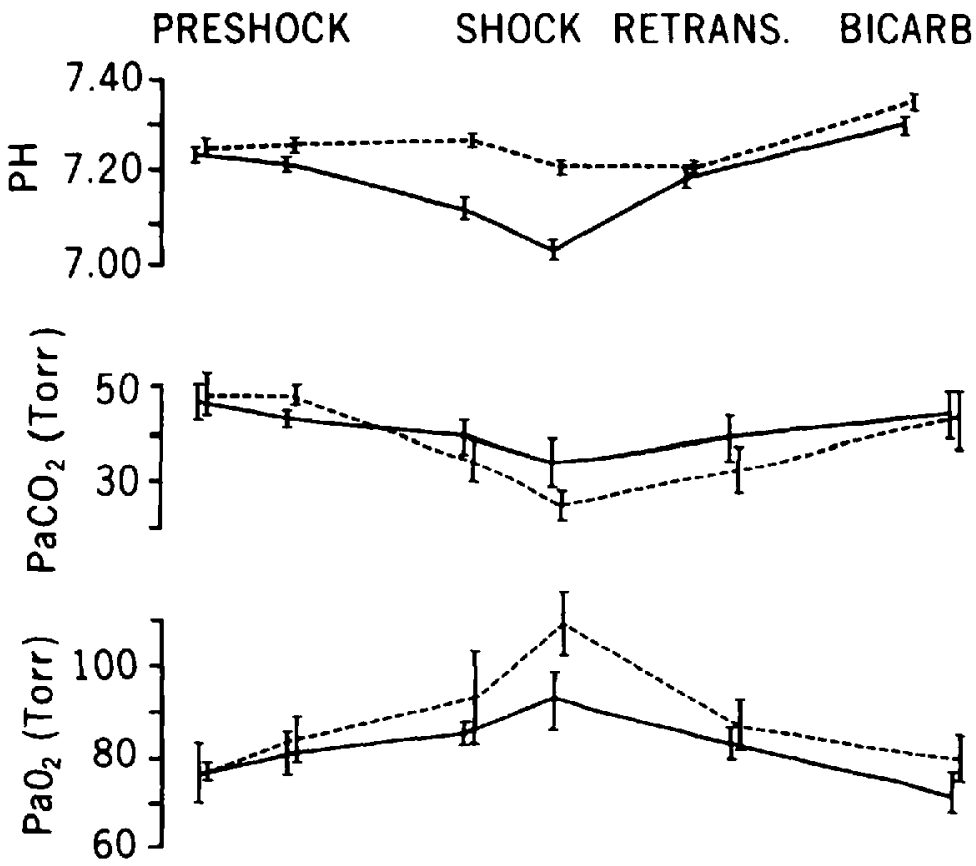

- CONTROL

Frgure 7. Blood gases in and out of shock in both control and chloroquine-treated dogs. 

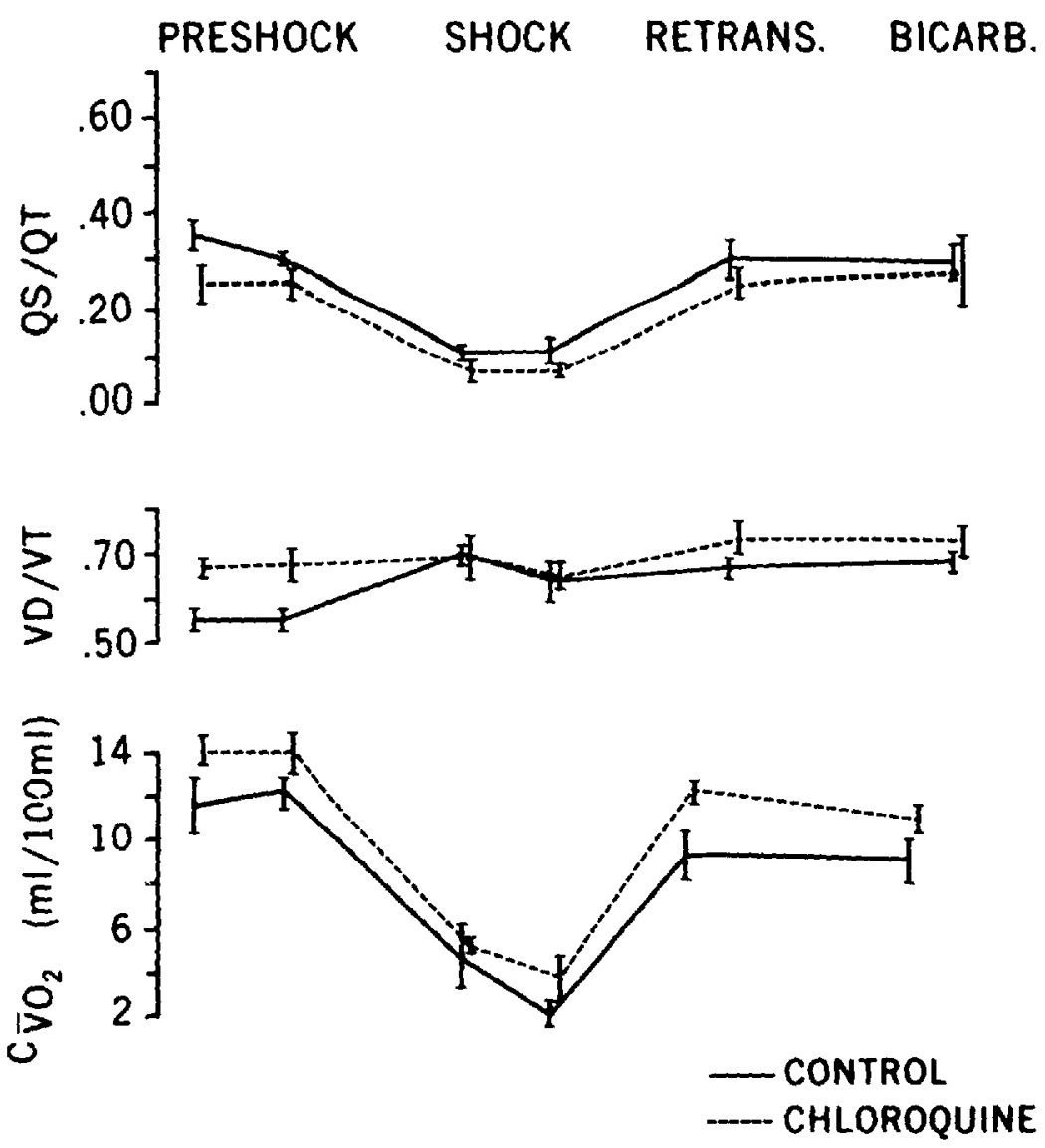

FIGURE 8. Changes in shunt (QS/QT), dead space (VD/VT) and central venous oxygen content $\left(\mathrm{CV}_{\mathrm{O}_{2}}\right)$.

\section{Drscussion}

The behaviour of the chloroquine-treated dogs in and out of shock differed from the control in several ways. Platelet aggregation was reduced by 50 per cent in the chloroquine group with a resultant decrease in platelet loss during shock (Figure 5).

Mean PA pressure returned to pre-shock value after bicarbonate administration in the chloroquine group but was still elevated by 33 per cent in the control group.

These results are similar to those obtained with aspirin though less marked. ${ }^{3}$ There was a significant decrease in lung water accumulation after shock in aspirintreated dogs but this is not observed with the chloroquine-treated dogs. Platelet aggregation was reduced 100 per cent in the aspirin-treated dogs but only 50 per cent in the chloroquine-treated dogs.

One of the earliest documented studies on the anti-aggregation effects of chloroquine was by Knisely ${ }^{4}$ and associates. They observed serial peripheral circulatory changes in monkeys infected with malaria before and after treatment with antimalarial drugs. Within 6 to 12 hours after treatment, a marked decrease in aggregation was observed. Linear blood flow velocity was increased, some plugged vessels 
became unplugged with resultant decreases in thrombotic and embolic phenomenon and a general clinical improvement of the treated animals. Subsequently a clinical study was done by Madow ${ }^{5}$ on 44 human subjects with various vascular diseases including coronary artery disease, cerebrovascular disease, thrombophlebitis, intermittent claudication, and one patient with sickle-cell anaemia. The average daily dose of chloroquine used was $600 \mathrm{mg}$. Marked clinical improvement was noted in these patients within a few days of therapy and discontinuation of treatment resulted in a prompt recurrence of symptoms.

More recently, Carter, et al. ${ }^{6}$ did a study on 565 patients during the 6th to 11th post-operative days. This period is associated statistically with the highest incidence of thrombotic and embolic episodes. One group was treated with intramuscular chloroquine starting from the operative day to two weeks post-operative. The average daily dose was chloroquine $600 \mathrm{mg}$. They repored a 9 per cent incidence of thrombosis in the untreated (control) group but none ( 0 per cent) in the chloroquine group. A 6 per cent incidence of pulmonary embolism was also reported in the control group while only 1 per cent incidence was observed in the chloroquine-treated patients. There was no increased bleeding associated with the use of chloroquine.

Our study on dogs subjected to haemorrhagic shock agrees with previous animal and human studies on the anti-aggregation effect of chloroquine. In this study, chloroquine produced a favourable effect on the pulmonary circulation. This effect was less marked than that obtained with the use of aspirin ${ }^{3}$ and less effective in reducing accumulation of water in the lung following shock.

The use of chloroquine deserves a closer second look in the prophylaxis and treatment of shock lung as well as other thrombotic and embolic states. One particular advantage of chloroquine is that it is not associated with increased bleeding. ${ }^{6}$ In areas of the world where malaria is endemic, chloroquine is a relatively common drug and its use can easily be extended to situations where its anti-platelet aggregation effect will be desirable without any risk of bleeding.

\section{SUMMARY}

One group of dogs received single doses of intramuscular chloroquine and another group served as a control. The dogs were subjected to haemorrhagic shock and then retransfused with their own blood after two hours of shock. The chloroquine-treated dogs had normal PA pressures after correction of acidosis and significantly smaller increases in the pulmonary vascular resistance. The results are similar but less marked than those obtained with aspirin. Chloroquine inhibited in vitro platelet aggregation by 50 per cent and was not associated with any increased bleeding. This may be an advantage when used in patients with multiple trauma or increased bleeding tendency.

\section{ACKNOWLEDGMENTS}

The expert technical assistance of Mr. Colin Kay, Mr. Gerry Carmichael, Mr. Tony DePersis and Mrs. Nancy Bell of the Anaesthesia Research Laboratory is 
gratefully acknowledged. We are also grateful to Ms. Susan Slack and Mrs. Carol Martin of the Haematology Research Laboratory, St. Michael's Hospital, for their co-operation.

This work was supported by the Medical Research Council of Canada grant \#MA 4263, and the Canadian Tuberculosis and Respiratory Disease Association.

Nembutal was supplied by Abbott Laboratories and the ACD blood packs by Baxter Laboratories.

\section{REFERENCES}

1. Noble, W.H. \& Severinghaus, J.W. Thermal and conductivity dilution curves for rapid quantitation of pulmonary edema. J. Applied Physiol. 32: 770 (1972).

2. Duncalf, Deryck \& Kuwabara, Shigeo. Computer analysis of respiratory data. Anaesthesia \& Analgesia: Current Researches 50: 5 (1971).

3. Famewo, C.E., Noble, W.H., \& Garvey, M.B. Use of aspirin in haemorrhagic shock. Can. Anaes. Soc. J. 22: 50 (1975).

4. KNisely, M.H., Stratman-Thomas, W.K., \& Eliot, T.S. Capillary circulation in malarial infected monkey: cinematographic study. J.A.M.A. 116: 2430 (1941).

5. MADOW, BENJAMIN P. Use of antimalarial drugs as "desludging" agents in vascular disease processes. J.A.M.A. 172: 1630 April (1960).

6. Carter, A.E., Eban, R., \& Perrett, R.D. Prevention of post-operative deep vein thrombosis and pulmonary embolism. British Medical Joumal 1: 312 (1971). 